\title{
INVESTIGATION OF MIXING IN TANKS OF A SPECIAL GEOMETRY
}

\author{
Bálint LeVente TarcsaY*1, Attila Egedy ${ }^{1}$, Janka BobeK², and Dóra Rippel-Pethő² \\ ${ }^{1}$ Department of Process Engineering, University of Pannonia, Egyetem u. 10, Veszprém, 8200, HUNGARY \\ ${ }^{2}$ Department of Chem. Eng. Sci.s, University of Pannonia, Egyetem u. 10, Veszprém, 8200, HUNGARY
}

\begin{abstract}
Mixing is one of the most crucial processes in the chemical industry. Homogeneity is a requirement for all feedstocks and industrial products. The degree of mixing depends on the hydrodynamic properties of the fluid in the units. The Residence Time Distribution (RTD) was investigated in a tank of a special geometry. Mixing was investigated using various geometries of the tank by applying the Heaviside function in step-response experiments. After obtaining experimental results, the RTD function was calculated. The flow structure in the tank was approximated by fitting black-box transfer function models onto the RTD function of the system. Two general model structures were defined and their fitness compared. By evaluating the fitted models, a relationship was established between the flow structure in the tank and its geometry.
\end{abstract}

Keywords: tanks with of special geometry, mixing, flow pattern, transfer function model

\section{Introduction}

In industrial processes, the thorough homogeneity of materials is a necessity to ensure the quality of the finished products and the safety of the production process. Therefore, various industrial-scale techniques for the homogenisation of materials of various densities and phases have been developed over the years. In the case of fluids, homogenization can be achieved through the application of mixers in a device. The optimal type and geometry of these stirred tanks can be approximated by specific models which take the properties of the materials to be mixed and the desired degree of homogeneity into consideration [1]. In the case of the homogenisation of large volumes of liquids (petrochemical and radiochemical industries), external recirculation can be more advantageous since it is considerably more inexpensive in terms of both installation and operation. The operation of stirred tanks has been thoroughly investigated by using mathematical and experimental methods, however, research on mixing achieved by external recirculation is lacking [2]. Optimization of the mixing process to achieve the most homogenous product possible in an external recirculation tank can only be achieved after the flow structure of the unit is known. The aim of this research was to analyze the flow structure in a tank of special geometry, moreover, to determine the connection between the flow structure and geometry of the tank.

This research forms the basis for future investigations where the flow structure and homogenization in a tank will be tested through an external recirculation mixing

\footnotetext{
*Correspondence: tarcsaybalint95@gmail.com
}

process. Mixing in stirred tanks can traditionally be analyzed by step-response experiments.

Step-response experiments are a widely accepted and simple way of determining the flow structure of process units [3]. During these experiments, the input of the system is a tracer fed according to the Heaviside function. The time evolution of the tracer concentration in the output of the tank holds information regarding the flow and mixing properties of the system. The Heaviside function is the integral of the Dirac delta function $\delta(t)$ :

$$
S(t)=\int_{-\infty}^{\infty} \delta(t) d t= \begin{cases}0 & \text { if } t<t^{*} \\ 1 & \text { if } t \geq t^{*}\end{cases}
$$

used as an input in pulse-response experiments [4]. The Heaviside function, $S(t)$, as a function of time takes on a value of 0 if the investigated time $t$ is less than the time of the tracer feed $t^{*}$ and a value of 1 during and after being fed.

After experimental data was collected on the response function of the system, the residence time distribution function (RTD) could be calculated. The RTD function approximates the probability that the residence time of the fed tracer agent is less than a specified residence time [4]. The RTD function, $F$, was calculated from

$$
F(t)=\frac{R_{t}-R_{t-1}}{R_{\text {steady }}-R_{0}},
$$

where $R_{\text {steady }}$ and $R_{0}$ are the values of the response function in the steady state and at the beginning of the experiment, respectively.

By analyzing the RTD curve in the studied unit, mixing and flow can be characterised and compared to the 


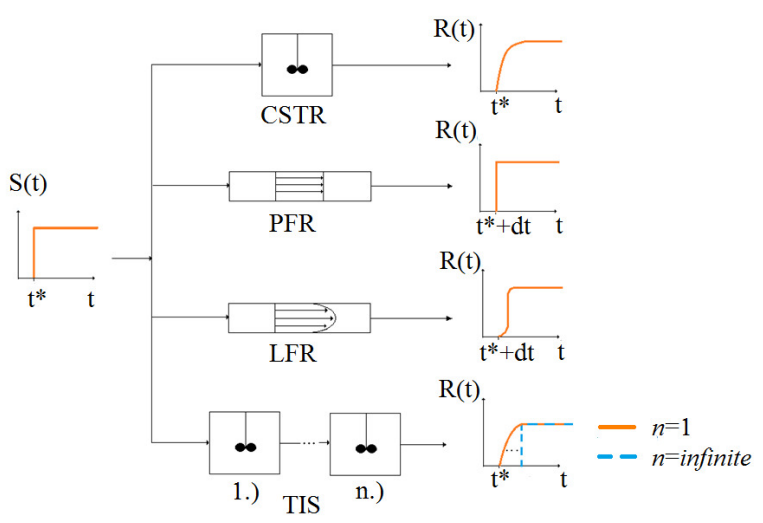

Figure 1: Response functions of various ideal flow models [3].

flow structure of ideal hydrodynamic models $[5,6]$. In this study, the ideal hydrodynamic models, which were used to describe the response, include the plug flow reactor (PFR) and tanks-in-series (TIS) model.

The PFR model is useful for modelling systems with a continuous flow of cylindrical geometry. It works under the assumption that the flow through a PFR is perfectly mixed in the radial direction but not in the axial direction. It is also assumed that the residence time of each fluid unit within the system is equal to the mean residence time, $\tau$. Because of these conditions, the step response function of a system with PFR tendencies is identical to the step function which was used as an input but with a time delay equal to the mean residence time in the unit. An altered version of the PFR model is the laminar flow reactor (LFR) model. The LFR model assumes a laminar flow structure within the system. Therefore, the residence time of the fluid phase within the system has a distribution. This causes the shape of the response function to become slightly altered compared to the step function. The TIS model is an extension of the continuous stirred tank reactor model (CSTR) which defines a system that is perfectly mixed and, therefore, homogeneous. Upon entering the unit, the tracer is dispersed throughout the whole tank, therefore, it immediately appears in the output without a time delay. The TIS model examines a series of CSTR units. The RTD function in this model is dependent on the number of tanks that have been linked. The RTD function of the TIS model converges towards the PFR model as the number of units increases and towards the CSTR distribution as the number of units decreases. One tank returns the RTD function of a CSTR and as the number of tanks in series approaches infinity, the RTD function converges towards the PFR model. The characteristic RTD curves of the aforementioned ideal flow models are shown in Fig. 1 [3]. In this figure, $t^{*}$ denotes the duration of tracer injection and $d t$ represents the time delay when the tracer appears in the outlet.

In this investigation, black-box models were used to approximate the behavior of the tank. Black- and whitebox modelling are two distinct modelling techniques to describe the traits of various systems. White-box models utilize well-known physical and chemical laws to characterize a system. These laws come in the form of differential equations, algebraic equations, etc. The model defines the internal functioning of the system and calculates the specific output from the input by taking these into consideration. Generally in the case of complex mixing problems, computational fluid dynamics (CFD) simulators are utilized to solve the white-box model of the system [7,8]. CFD simulations can determine the flow field in various units [9].

These simulators can be used to solve scientific problems from a diverse spectrum of disciplines, e.g. aerodynamics, hydrodynamics, ocean engineering, chemical engineering, etc. [10]. To solve these problems, various numerical methods have been invented over the years among which the finite element method (FEM) is one of the more frequently applied approaches [11]. However, in cases where the FEM is insufficiently advanced, alternative numerical methods can also be implemented, e.g. the spectral element method (SEM) [12]. Their disadvantage is, however, that since they model the systems so thoroughly, they require considerable computational power and computation time. Through these methods, it is possible to analyze processes which are more complex or require long experimentation times to be dissected. Despite this, these methods also require experimental data for validation.

Black-box models, on the other hand, provide generalized means for the modelling of various problems [13]. They often employ empirical methods to calculate the output of a system from the input without thoroughly describing the internal properties of the system [14]. The relationship between the input and output can be modelled using various techniques with the most popular being neural networks as well as state-space and transfer function models [15]. In this study, the transfer function model was utilized to approximate the behavior of a tank of special geometry.

\section{Experimental and the modelling pro- cess}

The geometry of the investigated tank is shown in Fig. 2. The tank consists of 5 cylindrical units integrated with each other. A removable wall was implemented which makes it possible to create the desired number of cylinders from the main tank. Therefore, the number of cylindrical units can be varied between 1 and 5 . During the investigation, geometries consisting of 1,3 and 5 cylindrical units were investigated. The residence time, calculated from the inlet volume flow rate and volume of the liquid, was $3 \mathrm{~h}$ for all geometries. Therefore, the inlet volume flow rate changed as the volume of liquid and number of cylindrical units varied. To obtain the response function of the system, a Heaviside function was used. The utilized tracer was a borax solution of low concentration $\left(6.24 \mathrm{gl}^{-1}\right)$. The tracer was fed into the tank which 


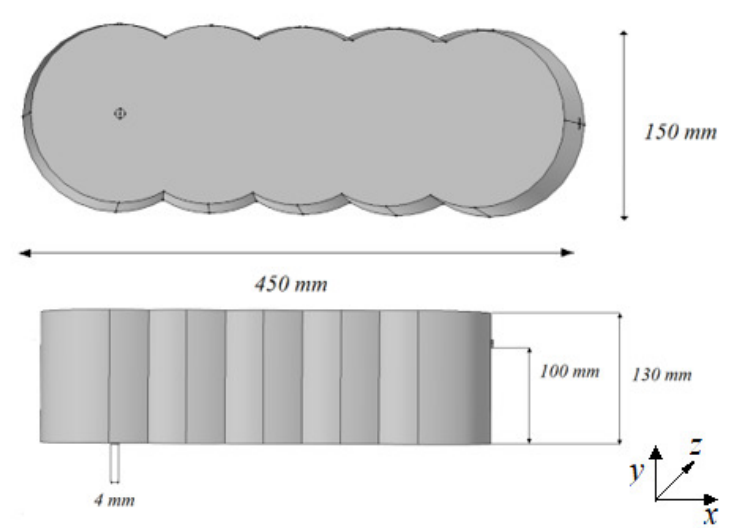

Figure 2: The investigated tank.

was filled with a high concentration of borax solution $\left(29.16 \mathrm{gl}^{-1}\right)$.

Conductivity measurements were used to monitor the change in concentration and obtain the response function. The experimental equipment is shown in Fig. 3. The experimental conditions for the feed and residence time are shown in Table 1.

The positions of the inlet and outlet during the experiments are shown in Table 2 . The $(0,0,0)$ coordinates of the system were defined as the bottom of the tank at the position of the outlet. The coordinates $x, y$ and $z$ correspond to the longitudinal, vertical and horizontal distances, respectively.

The RTD curves of the three different geometries of the tank were investigated. After experimentally obtaining the data from the RTD curves, transfer function models were fitted to the experimental results. Two types of fitted functions were investigated. Both were black-box convolution models of the PFR and TIS. However, one contained only serial links between the perfectly mixed units, the other assumed parallel connections as well. Differential equations describe the change in concentration of the output of the tank as a function of the input. In various ideal flow models, these equations were converted into algebraic equations by using the Laplace transform. After this the transfer function of the ideal flow mod-

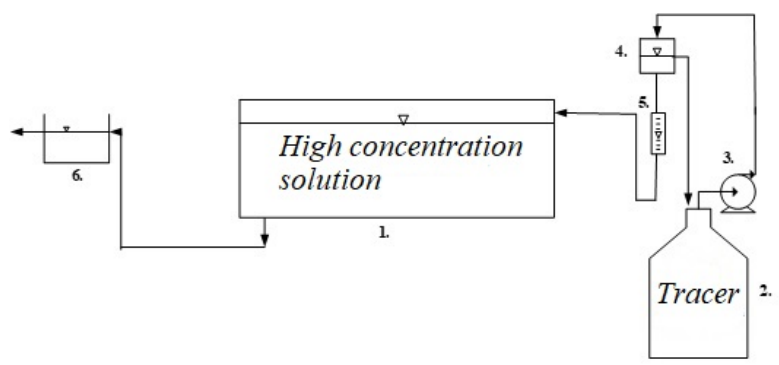

Figure 3: Setup of the experimental equipment (1experimental tank, 2-tracer containment tank, 3peristaltic pump, 4-buffer unit, 5-rotameter, 6-output containment tank).
Table 1: Experimental parameters.

\begin{tabular}{c|c|c}
\hline Number of Cylinders & $\tau(\mathrm{h})$ & $B\left(\mathrm{lh}^{-1}\right)$ \\
\hline 1 & & 0.4 \\
3 & 3 & 1.1 \\
5 & & 1.7 \\
\hline
\end{tabular}

Table 2: Inlet and outlet positions.

\begin{tabular}{c|ccc|ccc}
\hline $\begin{array}{c}\text { Number of } \\
\text { Cylinders }\end{array}$ & \multicolumn{3}{|c|}{ Inlet position } & \multicolumn{3}{c}{ Outlet position } \\
\hline & $\begin{array}{c}\mathrm{x} \\
(\mathrm{mm})\end{array}$ & $\begin{array}{c}\mathrm{y} \\
(\mathrm{mm})\end{array}$ & $\begin{array}{c}\mathrm{z} \\
(\mathrm{mm})\end{array}$ & $\begin{array}{c}\mathrm{x} \\
(\mathrm{mm})\end{array}$ & $\begin{array}{c}\mathrm{y} \\
(\mathrm{mm})\end{array}$ & $\begin{array}{c}\mathrm{Z} \\
(\mathrm{mm})\end{array}$ \\
\hline 1 & 75 & 100 & 0 & 0 & 2 & 0 \\
3 & 225 & 100 & 0 & 0 & 2 & 0 \\
5 & 375 & 100 & 0 & 0 & 2 & 0 \\
\hline
\end{tabular}

els was defined as the ratio of the output (the Laplace transformed response function) to the input of the system (the step function of the tracer injection). If the product of multiple elementary transfer functions is computed, a TIS model can be generated. A general model structure for this network can be seen in Fig. 4.

On the other hand, by combining the elementary transfer functions of ideal flow models, a network of flow models with parallel links can be created. A general model for this structure is shown in Fig. 5. In both cases, a PFR unit has been included in the structure, in general this is useful for modelling systems with a time delay. The time delay in the RTD function could point to either PFR tendencies in the flow structure or the presence of dead volumes within the tank. To distinguish which is present, further investigations with a CFD simulator are required.

The general form of the transfer function that only uses serial connections is

$$
G_{\text {fitted }}=\frac{a}{b_{n} s^{n}+b_{n-1} s^{n-1}+\cdots+b_{0} s^{0}} e^{-t_{\mathrm{d}} s},
$$

where $n$ denotes the order of the denominator and $b_{n}, a$, and $t_{d}$ are parameters of the transfer function.

In the case of the model containing both parallel and serial links, the general transfer function is

$$
G_{\text {fitted }}=\frac{a_{n-1} s^{n-1}+\cdots+a_{0} s^{0}}{b_{n} s^{n}+b_{n-1} s^{n-1}+\cdots+b_{0} s^{0}} e^{-t_{d} s},
$$

where $n$ is the order of the denominator and $b_{n}, a_{n}$, and $t_{d}$ are parameters of the transfer function. However, due to the parallel connections, the elementary transfer functions are not just multiplied but totalled. Therefore, the

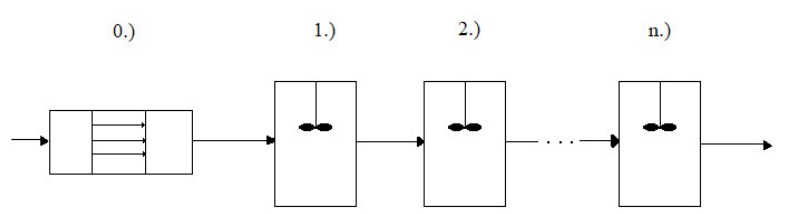

Figure 4: General model structure involving only serial links $[5,6]$. 


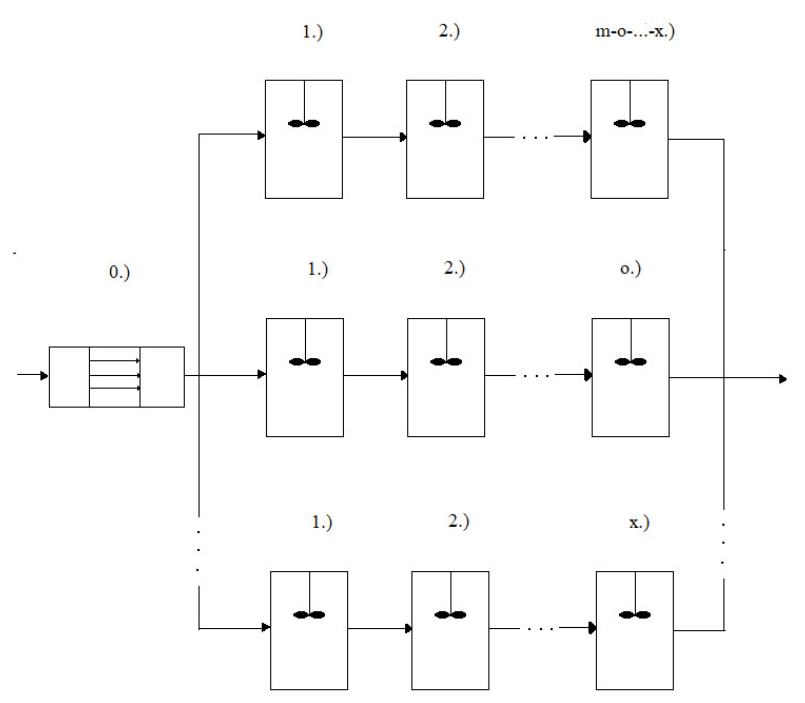

Figure 5: General model structure involving both serial and parallel links $[5,6]$.

nominator also contains a higher order polynomial. The order of this polynomial can be one at most below the order of the denominator for the system to be suitable.

In both cases, the fitted RTD curve $F_{\text {fitted }}$ was calculated from the step response of the system which was characterized by the transfer function, $G_{\text {fitted }}$.

The overall parameters of the transfer function were determined by numerically solving a minimization problem. The minimization problem is expressed in

$$
\min [E(n, a, b, d)]=\frac{\sum\left[F_{\text {exp }}-F_{\text {fitted }}(n, a, b, d)\right]^{2}}{\sum\left(F_{\text {exp }}\right)^{2}}
$$

where $E$ is a function denoting the sum of the squared difference of the experimental and fitted RTD functions divided by the sum of the square of the experimental RTD curve. As such, $E$ is a function similar to the relative error of the fitting which is dependent on the parameters of the transfer function, $G_{\text {fitted }}$.

Inversely, the goodness of the fit, $I$, can be defined as the complementer of the fitting error:

$$
I(n, a, b, d)=1-E(n, a, b, d) .
$$

For the minimization of the function, the interior-point method was applied in MATLAB R2011. Constraints were defined for the lower and upper boundaries of the parameters, namely 0 and 100 for all parameters, respectively. The fitting algorithm was modified to favor transfer functions with the smallest possible order and smallest relative error. The algorithm can be seen in Fig. 6 .

The algorithm follows a general route for fitting both parallel and serial models. During the first step, a loworder (first-order) transfer function is created. After that the parameters of this transfer function are identified using the interior point method. After obtaining the parameters, the $E$ function is calculated to evaluate the error of the fitting. If the error is less than a predefined threshold
$\left(E r r_{\text {opt }}\right)$, then the fitting will stop and the current transfer function be defined as the best possible fit. If the error of the fit exceeds the defined threshold, then the fitting continues. In this case, the order of the denominator is increased and the error of the higher order transfer function evaluated as well. If the error of fitting is less than the lower order transfer function, then the fitting continues until either a transfer function which satisfies the error boundary is identified or the error of the fitting does not decrease significantly even though the order of the denominator increases.

In the case of models which include parallel links, this algorithm contains an additional iteration step. During this step, the algorithm investigates the fit of an $n$th order transfer function (if $n \geq 2$ ) with varying orders of the nominator. To achieve this, the fitting is evaluated by applying increasing orders of the nominator. During this process, either the maximum order of the nominator $(n-1)$ is reached or a point where the increase in the order of the nominator is identified that does not significantly impact the fitting error. In either case, fitting is stopped and the solution accepted as the best possible fit for a transfer function with order of the denominator, $n$.

To evaluate the proposed fitting algorithm for both models, a custom-defined transfer function was analyzed. This reference transfer function $\left(G_{\text {ref }}\right.$ is defined by

$$
G_{\mathrm{ref}}=\frac{1}{1.6 s^{2}+2.6 s+1} e^{-5 s}
$$

in the case of the model contains only serial links.

The step response function for this transfer function was used as reference data. The extrema of function $E$ were numerically determined by using the devised algorithm. The fitted transfer function is

$$
G_{\mathrm{ref}}=\frac{1}{1.58 s^{2}+2.59 s+1} e^{-5 s} .
$$

Fig. 7 shows the fitting of the RTD of the identified transfer function to the reference RTD function. The goodness of the fit was $98.2 \%$.

The testing was also conducted in cases where parallel links are present besides serial links. In this case, the reference system is characterized by the transfer function:

$$
G_{\mathrm{ref}}=\frac{8.6 s+1}{s^{4}+2.5 s^{3}+11 s^{2}+10 s+1} e^{-2 s} .
$$

The fitting algorithm was augmented to identify both the optimal orders of the nominator and denominator. The fitted transfer function is

$$
G_{\text {fitted }}=\frac{8.95 s+1}{s^{4}+2.37 s^{3}+11.14 s^{2}+10.33 s+1} e^{-2 s} \text {. }
$$

The results of the fitting are displayed in Fig. 8. In this case, the goodness of the fit exceeded $99 \%$.

Both Fig. 8 and the measurements of the parameters show that the algorithm is capable of precisely fitting both models, therefore, it is suitable for identifying the transfer functions of the system. After validating the fitting 


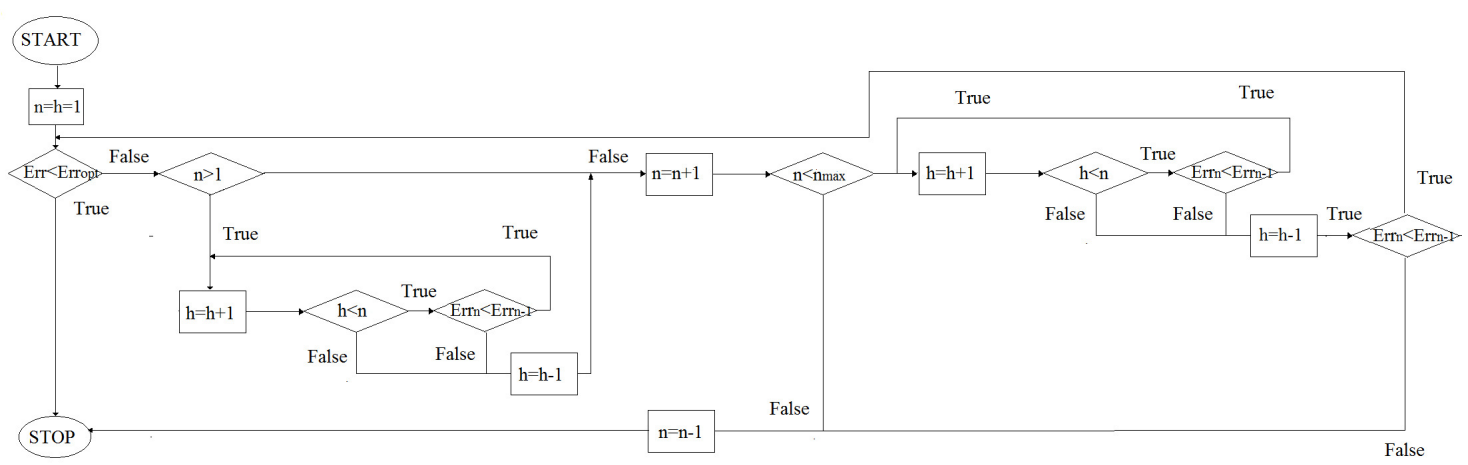

Figure 6: The general applied fitted algorithm for a model containing both serial and parallel links.

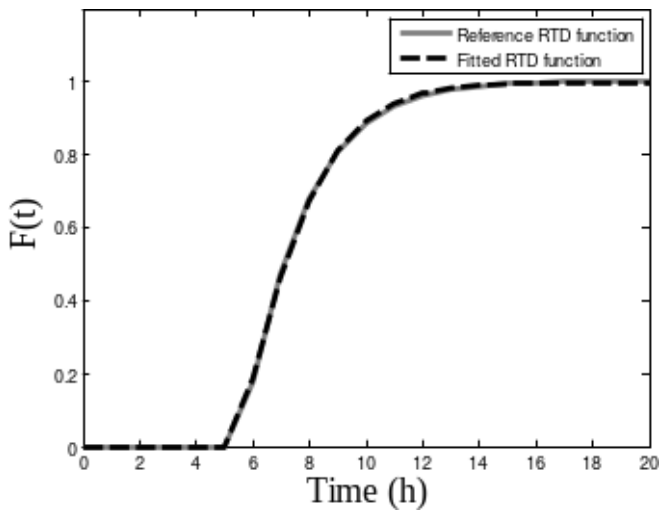

Figure 7: The results of the algorithm validation for fitting the model containing only serial connections.

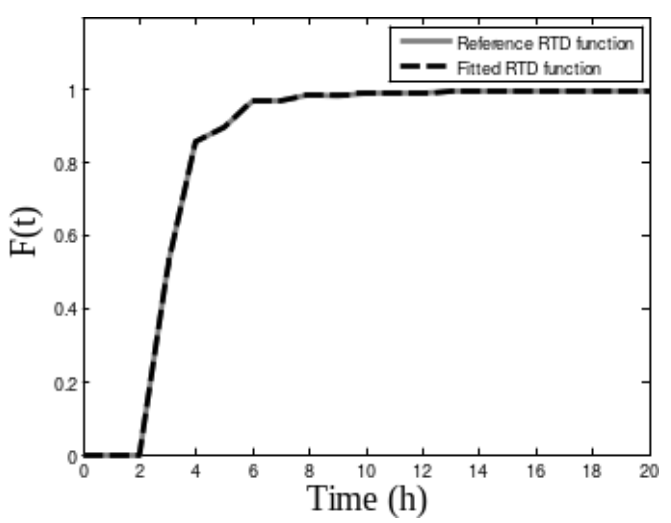

Figure 8: The results of the algorithm validation for fitting the model containing parallel and serial links.

method, transfer functions were identified for the tank by using the experimental RTD curves.

\section{Results and Analysis}

The results of the fittings in the case of the model with only serial connections can be seen in Figs. 9-11 for all three geometries of the tank that were investigated.

The geometry consisting of 5 cylinders was originally modeled using a second order transfer function that yielded the best fit for the experimental RTD curve (92 $\%)$. However, to compare the parameters of the fitting

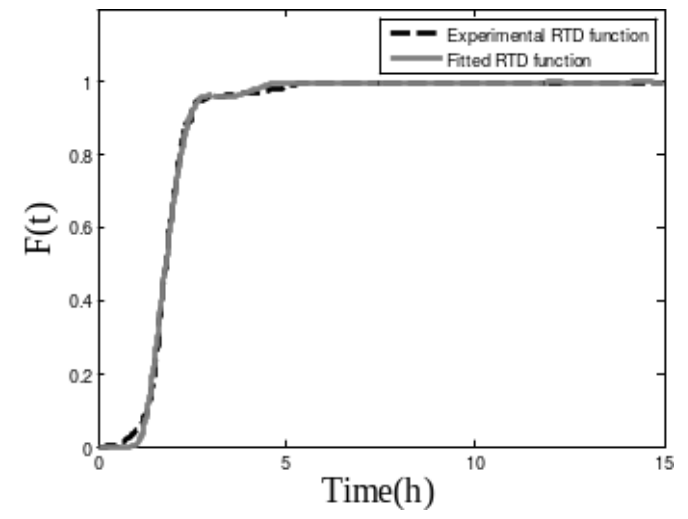

Figure 9: Results of the fitting of the serial model for 1 cylindrical unit.

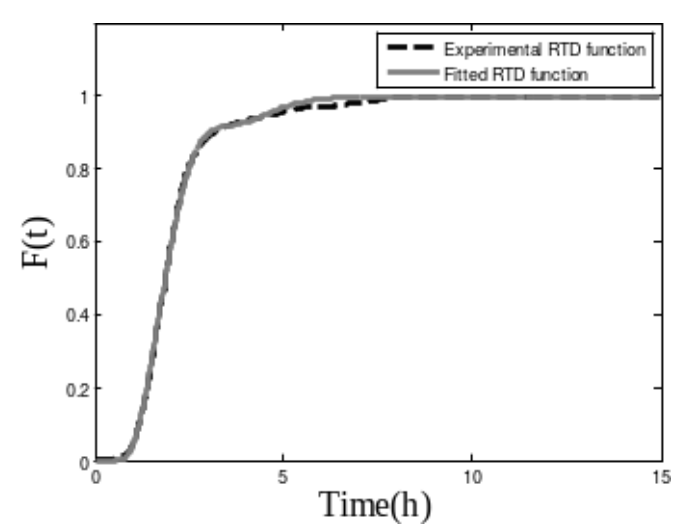

Figure 10: Results of the fitting of the serial model for 3 cylindrical units.

and characterise the system, it was more favorable to fit a third order transfer function here as well since it describes the other two RTD curves best. In this case, the average goodness of the fit was $91 \%$ with the goodness of the fit in the case of the geometry consisting of 5 cylindrical units $(86 \%)$ being the lowest. The numerical parameters of the fit are shown in Table 3.

The results are as follows: to describe the system in the cases of 1 and 3 cylindrical units, a third order transfer function was used. In the case of 5 cylindrical units, a second order transfer function was optimal.

In the case of the model containing both parallel and 


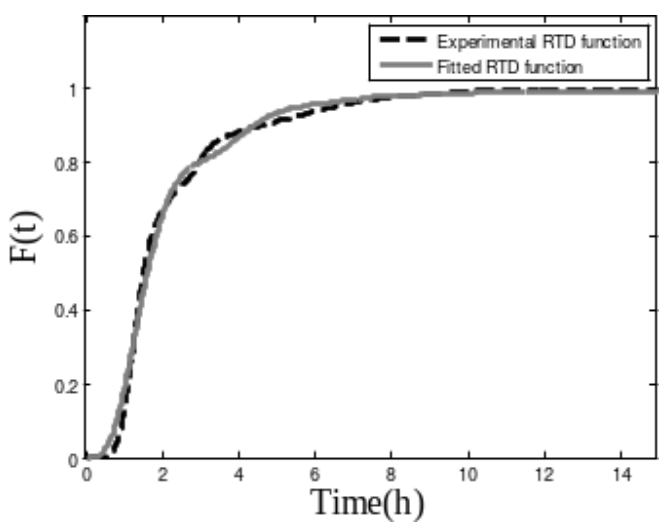

Figure 11: Results of the fitting of the serial model for 5 cylindrical units.

Table 3: Fitted parameters for the model containing only serial links.

\begin{tabular}{c|cc|cccc|cc}
\hline \multicolumn{10}{c}{ Parameters } \\
\hline$N$ & $n$ & $a_{0}$ & $b_{3}$ & $b_{2}$ & $b_{1}$ & $b_{0}$ & $t_{d}(\mathrm{~h})$ & $I(\%)$ \\
\hline 1 & 3 & 1 & 0.1 & 0.3 & 1.0 & 1 & 0.9 & 93.43 \\
3 & 3 & 1 & 0.2 & 0.6 & 1.5 & 1 & 0.6 & 94.00 \\
5 & 3 & 1 & 0.3 & 0.7 & 2.0 & 1 & 0.7 & 85.76 \\
5 & 2 & 1 & - & 0.9 & 2.3 & 1 & 0.7 & 92.00 \\
\hline
\end{tabular}

serial links, the fitted curves are displayed in Figs. 12-14.

In the case of the model containing both parallel and serial connections, the tendencies are similar to the results of the fitting conducted using the model consisting of only serial links. However, the goodness of the fit was enhanced compared to the simpler models (over $99 \%$ for all investigated geometries). In this case, the behavior of the tank consisting of 1 cylindrical unit was described by a transfer function where the orders of the nominator and denominator were 4 and 2, respectively. In the case of 3 and 5 cylindrical units, the orders of the denominator and nominator were 3 and 1 , respectively. The order of the transfer function (the number of CSTR units in the TIS model) increases as the number of cylinders decreases. This is evident of an increase in the PFR/LFR tendencies of the functions which can also be seen in the fig-

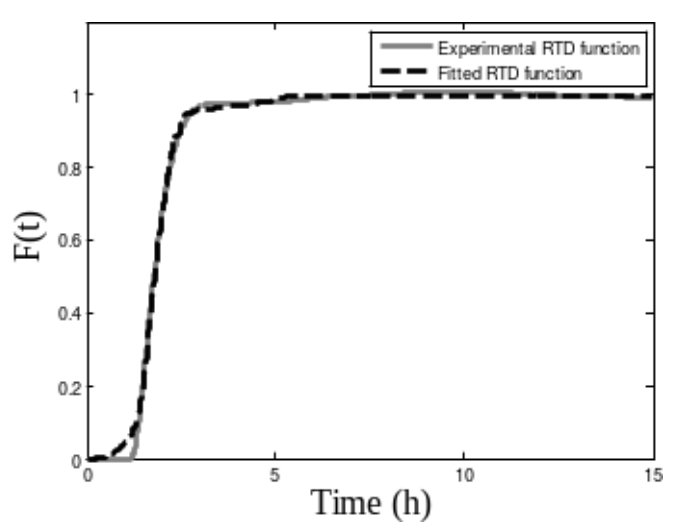

Figure 12: Results of the fitting of the parallel model for 1 cylindrical unit.

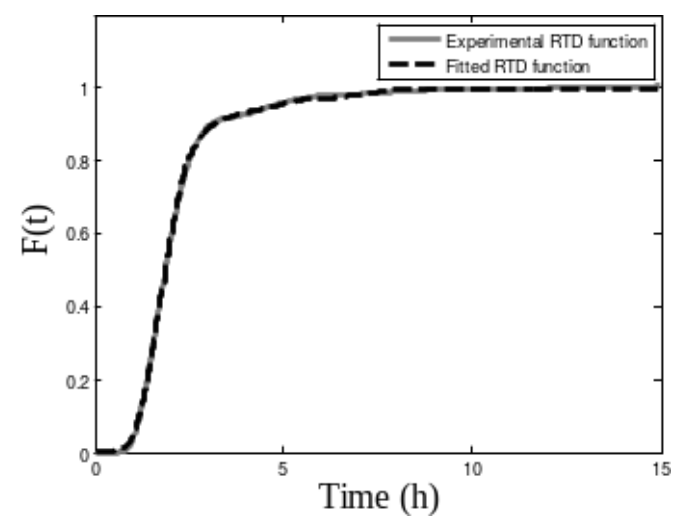

Figure 13: Results of the fitting of the parallel model for 3 cylindrical units.

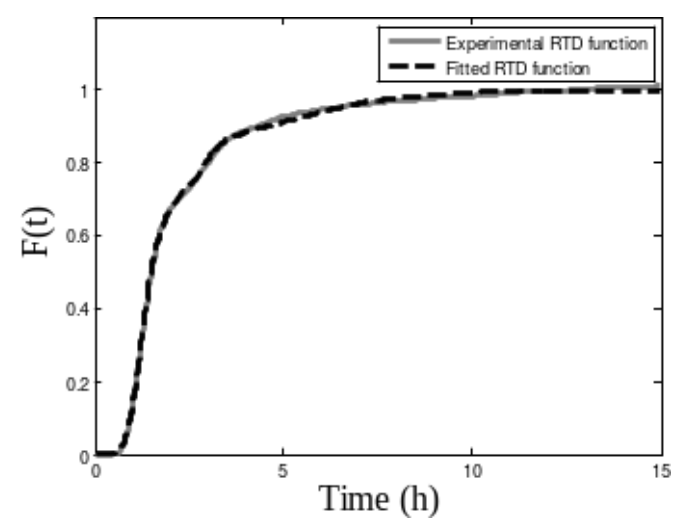

Figure 14: Results of the fitting of the parallel model for 5 cylindrical units.

ures. It should also be noted, however, that the fitting in all models was exceedingly high (over $95 \%$ on average). This might suggest that the model algorithm is too precise and the model was also fitted according to the experimental noise remaining in the data. In the absence of the noise, such tendencies might be clearer and the decrease in the order of the nominator between the different geometries more prominent. Further investigations carried out by CFD simulations may clarify these concerns.

The parameters of the fit are displayed in Table 4.

\section{Conclusion}

In this paper, the velocity field of a tank of special geometry was investigated. In order to determine the relationship between the flow profile and geometry of the tank, step-response experiments were conducted. The RTD curves of the system were measured by applying various geometries of the tank. By analyzing the experimental RTD curves, a model was proposed to be fitted onto the results. The transfer function of the system was optimized by minimalizing the squared difference of the experimental and fitted RTD curves. The interior-point method was applied to minimalize the difference. The following conclusions were drawn from the fitted RTD curves: 
Table 4: Fitted parameters of the model containing both parallel and serial links.

\begin{tabular}{c|cc|ccc|ccccc|cc}
\hline \multicolumn{10}{c}{ Parameters } \\
\hline$N$ & $n$ & $m$ & $a_{2}$ & $a_{1}$ & $a_{0}$ & $b_{4}$ & $b_{3}$ & $b_{2}$ & $b_{1}$ & $b_{0}$ & $t_{d}(\mathrm{~h})$ & $I(\%)$ \\
\hline 1 & 4 & 2 & 6 & 2.4 & 1 & 1 & 4.3 & 8 & 3.12 & 1 & 1.2 & 98.28 \\
3 & 4 & 1 & - & 5.5 & 1 & 1 & 3.4 & 8.5 & 7.1 & 1 & 0.6 & 99.77 \\
5 & 3 & 1 & - & 12 & 1 & 1 & 3.5 & 18 & 14.4 & 1 & 0.5 & 99.43 \\
\hline
\end{tabular}

In the case of models containing only serial links, 1 and 3 cylindrical units could be optimally described using a third order transfer function. The geometry consisting of 5 cylindrical unitscan be described by a second order transfer function. The average goodness of the fit in this case was $91 \%$. In the case of the model utilizing parallel links, the RTD function of the tank consisting of 1 cylindrical unit was approximated by a transfer function where the orders of the denominator and nominator were 4 and 2, respectively. In the cases of 3 and 5 cylindrical units, the orders of the denominator and nominator were 3 and 1, respectively. The goodness of fit on average in the case of this model was over $99 \%$.

It can be concluded that all geometries of the tank can be described using fourth order or lower transfer functions with increasing model parameters according to the number of cylinders.

Further investigations to characterise the reasons for such behavior will be carried out by using CFD methods. Simultaneously, different step-response experiments will be conducted to observe the behavior of the system. During these experiments, instead of feeding the solution of lower concentration into that of higher concentration within the tank, a positive step function will be used. As a result, the orders will be reversed with the solution of low concentration being in the tank and that of high concentration in the feed.

\section{Notations}

\section{Latin letters}

a nominator parameter of transfer function

$b$ denominator parameter of transfer function

$d t \quad$ time delay [h]

$h \quad$ order of the nominator of transfer function $(1, n-1)$

$n \quad$ order of the denominator of transfer function [-]

$t$ time [h]

$t_{d} \quad$ time delay of transfer function [h]

$x \quad$ longitudinal coordinate [mm]

$y \quad$ vertical coordinate [mm]

$z \quad$ horizontal coordinate [mm]

\section{Capital letters}

$B$ volume flow rate $\left[\mathrm{lh}^{-1}\right]$

$E$ error of fitting [\%]

$F$ residence time distribution function

$G \quad$ transfer function

I goodness of fit [\%]

$N \quad$ number of cylinders

$R \quad$ response function $\left[\mathrm{gl}-{ }^{1}\right]$

$S \quad$ step function $\left[\mathrm{gl}^{-1}\right]$

\begin{tabular}{ll}
$\delta$ & \multicolumn{2}{c}{ Greek letters } \\
$\tau$ & Dirac delta function \\
mean residence time $[\mathrm{h}]$
\end{tabular}

\section{Acknowledgement}

This research was supported by the project EFOP-3.6.116-2016-00015 "Smart Specialisation Strategy (S3) comprehensive institutional development program at the University of Pannonia to promote sensible individual education and career choices".

\section{REFERENCES}

[1] Nienow, A. W., Edwards, M. F., Harnby, N. Mixing in the Process Industries. (Elsevier, Oxford, United Kingdom 1997) ISBN: 0- 7506-3760-9

[2] Blais, B., Lassaigne, M., Goniva, C., Fradette, L., Bertrand, F. Development of an unresolved CFD-DEM model for the flow of viscous suspensions and its application to solid-liquid mixing. J. Comp. Phys., 2016, 318, 201-221 DOI: 10.1016/j.jcp.2016.05.008

[3] Levenspiel, O. Chemical reaction engineering. Ind. Eng. Chem. Res., 1999, 38(11), 4140-4143 DOI: 10.1021/ie990488g

[4] Danckwerts, P. V. Continuous flow systems: Distribution of residence times. Chem. Eng. Sci., 1995, 50(24), 3857-3866 DOI: 10.1016/0009-2509(96)81811-2

[5] Fazli-Abukheyli, R., Darvishi, P. Combination of axial dispersion and velocity profile in parallel tanks-in-series compartment model for prediction of residence time distribution in a wide range of non-ideal laminar flow regimes. Chem. Eng. Sci., 2019, 195, 531-540 DOI: 10.1016/j.ces.2018.09.052

[6] Haag, J., Gentric, C., Lemaitre, C., Leclerc, J. P. Modelling of Chemical Reactors: From Systemic Approach to Compartmental Modelling. Int. J. Chem. Reactor Eng., 2018, 16(8), 1-22 DOI: 10.1515/ijcre-2017-0172

[7] Blazek, J. Computational Fluid Dynamics: Principles and Applications. (Butterworth-Heinemann, 
Oxford, United Kingdom 2015) ISBN: 978-0-08099995-1

[8] Toro, E. F. Riemann Solvers and Numerical Methods for Fluid Dynamics. (Springer, Heidelberg, Germany 1999) ISBN: 978-3-662-03492-7

[9] Pawlowski, S., Nayak, N., Meireles, M., Portugal, C. A. M., Velizarov, S., Crespo, J. G. CFD modelling of flow patterns, tortuosity and residence time distribution in monolithic porous columns reconstructed from X-ray tomography data. Chem. Eng. J., 2018, 350, 757-766 DOI: 10.1016/j.cej.2018.06.017

[10] Delafosse, A., Collignon, M. L., Calvo, S., Delvigne, F., Crine, M., Thonart, P., Toye, D.: CFD-based compartment model for description of mixing in bioreactors. Chem. Eng. Sci., 2014, 106, 76-85 DOI: 10.1016/j.ces.2013.11.033

[11] Zienkiewicz, O. C., Taylor, R. L., Zhu, J. Z. The Fi- nite Element Method: Its Basis and Fundamentals. (Elsevier, Oxford, United Kingdom, 2005) ISBN: 07506-6320-0

[12] Solin, P., Segeth, K., Dolezel, I. Higher-Order Finite Element Methods. (Chapman and Hall/CRC, New York, 2003) ISBN: 978-0-42-920527-9

[13] Witt, C., Bux, M., Gusew, W., Leser, U. Predictive performance modeling for distributed batch processing using black box monitoring and machine learning. Information Systems 2019, 82, 33-52 DOI: 10.1016/j.is.2019.01.006

[14] Suykens, Johan A. K.; Vandewalle, Joos P. L. (ed.) Nonlinear Modelling: Advanced Black-Box Techniques. (Springer Science and Business Media, Leuven, 2012) ISBN: 978-1-4613-7611-8

[15] Bunge, M. A general black box theory. Philosophy of Science, 1963, 30(4), 346-358 DOI: 10.1086/287954 